\title{
Controlling electron-electron correlation in frustrated double ionization of triatomic molecules with orthogonally polarized two-color laser fields
}

\author{
A. Chen, ${ }^{1}$ M. F. Kling, ${ }^{2,3}$ and A. Emmanouilidou ${ }^{1}$ \\ ${ }^{1}$ Department of Physics and Astronomy, University College London, Gower Street, London WC1E 6BT, United Kingdom \\ ${ }^{2}$ Department of Physics, Ludwig-Maximilians-Universität Munich, Am Coulombwall 1, D-85748 Garching, Germany \\ ${ }^{3}$ Max Planck Institute of Quantum Optics, Hans-Kopfermann-Strasse 1, D-85748 Garching, Germany
}

(Received 26 June 2017; published 6 September 2017)

\begin{abstract}
We demonstrate the control of electron-electron correlation in frustrated double ionization (FDI) of the two-electron triatomic molecule $\mathrm{D}_{3}{ }^{+}$when driven by two orthogonally polarized two-color laser fields. We employ a three-dimensional semiclassical model that fully accounts for the electron and nuclear motion in strong fields. We analyze the FDI probability and the distribution of the momentum of the escaping electron along the polarization direction of the longer wavelength and more intense laser field. These observables, when considered in conjunction, bear clear signatures of the prevalence or absence of electron-electron correlation in FDI, depending on the time delay between the two laser pulses. We find that $\mathrm{D}_{3}{ }^{+}$is a better candidate than $\mathrm{H}_{2}$ for demonstrating also experimentally that electron-electron correlation indeed underlies FDI.
\end{abstract}

DOI: 10.1103/PhysRevA.96.033404

\section{INTRODUCTION}

Frustrated double ionization (FDI) is a major process in the nonlinear response of multicenter molecules when driven by intense laser fields, accounting for roughly $10 \%$ of all ionization events [1,2]. In frustrated ionization an electron first tunnel ionizes in the driving laser field. Then, due to the electric field of the laser pulse, it is recaptured by the parent ion in a Rydberg state [3]. This process is a candidate for the inversion of $\mathrm{N}_{2}$ in free-space air lasing [4]. In FDI an electron escapes, and another one occupies a Rydberg state at the end of the laser pulse. FDI has attracted considerable interest in recent years in a number of experimental studies in the context of $\mathrm{H}_{2}$ [1] and of the triatomic molecules $\mathrm{D}_{3}{ }^{+}$and $\mathrm{H}_{3}{ }^{+}$[5-7].

In theoretical studies of strongly driven two-electron diatomic and triatomic molecules, two pathways of FDI have been identified [2,8]. Electron-electron correlation is important, primarily, for one of the two pathways. It is well accepted that electron-electron correlation underlies a significant part of double ionization in strongly driven molecules, a mechanism known as nonsequential double ionization $[9,10]$. However, electron-electron correlation in FDI has yet to be accessed experimentally.

Here, we propose a road for future experiments to identify the important role of electron-electron correlation in FDI. We identify the parameters of orthogonally polarized two-color (OTC) laser fields that best control the relevant pathway for electron-electron correlation in FDI. We demonstrate traces of attosecond control of electron motion in space and time in two observables of FDI as a function of the time delay between the fundamental 800-nm and second-harmonic 400-nm laser fields. We show that, together, the FDI probability and the momentum of the escaping electron along the fundamental laser field bear clear signatures of the turning on and off of electron-electron correlation.

Two-color laser fields are an efficient tool for controlling electron motion [11-13] and for steering the outcome of chemical reactions [14-16]. Other applications include the field-free orientation of molecules [17-19], the generation of high-harmonic spectra [20-23], and probing atomic and molecular orbital symmetry [24-26].

The strongly driven dynamics of two electrons and three nuclei poses a challenge for fully $a b$ initio quantum-mechanical calculations. The latter techniques can currently address one-electron triatomic molecules [27]. Therefore, we rely on classical and semiclassical models for understanding the fragmentation dynamics in triatomic molecules driven by intense infrared laser pulses [8,28]. Our work employs a threedimensional semiclassical model. This model has provided significant insights into FDI for strongly driven $\mathrm{H}_{2}$ [2] and $\mathrm{D}_{3}{ }^{+}$[8]. Our previous result for the distribution of the kinetic energy release of the Coulomb exploding nuclei in FDI of $\mathrm{D}_{3}{ }^{+}$ was in good agreement with experiment [7].

\section{METHOD}

We employ the initial state of $\mathrm{D}_{3}{ }^{+}$that is accessed experimentally via the reaction $\mathrm{D}_{2}+\mathrm{D}_{2}{ }^{+} \rightarrow \mathrm{D}_{3}{ }^{+}+\mathrm{D}[5,7]$. It consists of a superposition of triangular-configuration vibrational states $v=1-12$ [7,29]. We assume that most of the $\mathrm{D}_{3}^{+}$ionization occurs at the outer classical turning point of the vibrational levels $[30,31]$. The turning point varies from 2.04 a.u. $(v=1)$ to 2.92 a.u. $(v=12)[29,32]$. We initialize the nuclei at rest for all vibrational levels since an initial predissociation does not significantly modify the ionization dynamics [33].

The combined strength of the two laser fields is within the below-the-barrier ionization regime. To formulate the initial state of the two electrons, we assume that one electron (electron 1) tunnel ionizes at time $t_{0}$ in the field-lowered Coulomb potential. For this quantum-mechanical step, we compute the ionization rate using a semiclassical formula [34]. $t_{0}$ is selected using importance sampling [35] in the time interval in which the two-color laser field is present. The ionization rate is then used as the importance sampling distribution. For electron 1 , the velocity component that is transverse to the OTC laser fields is given by a Gaussian [36], and the component that is parallel is set equal to zero. 
The initial state of the initially bound electron (electron 2) is described by a microcanonical distribution [37].

Another quantum-mechanical aspect of our threedimensional model is the tunneling of each electron during the propagation with a probability given by the Wentzel-KramersBrillouin approximation [2,33]. This aspect is essential to accurately describe the enhanced ionization (EI) process $[10,38]$. In EI, at a critical distance of the nuclei, a double-potential well is formed such that it is easier for an electron bound to the higher potential well to tunnel to the lower potential well and subsequently ionize. The time propagation is classical, starting from time $t_{0}$. We solve the classical equations of motion for the Hamiltonian of the strongly driven five-body system while fully accounting for the Coulomb singularities [33].

The OTC laser field we employ is of the form

$$
\begin{aligned}
\mathbf{E}(t, \Delta t)= & E_{\omega} f(t) \cos (\omega t) \hat{z} \\
& +E_{2 \omega} f(t+\Delta t) \cos [2 \omega(t+\Delta t)] \hat{x}, \\
f(t)= & \exp \left[-2 \ln 2\left(\frac{t}{\tau_{F W H M}}\right)^{2}\right],
\end{aligned}
$$

with $\omega=0.057$ a.u. for commonly used Ti:sapphire lasers at $800 \mathrm{~nm}$. $T_{\omega}$ and $T_{2 \omega}$ are the corresponding periods of the fundamental and second-harmonic laser fields, polarized along the $\hat{z}$ and $\hat{x}$ axes, respectively. $\tau_{F W H M}=40$ fs is the FWHM. $\Delta t$ is the time delay between the $\omega$ and $2 \omega$ pulses. We consider $E_{\omega}=0.08$ a.u. since for this field strength pathway B of FDI, where electron-electron correlation is present, prevails over pathway A: $4.8 \%$ versus $3.6 \%$ [8].

In FDI of $\mathrm{D}_{3}{ }^{+}$the final fragments are a neutral excited fragment $\mathrm{D}^{*}$, two $\mathrm{D}^{+}$ions, and one escaping electron. In the neutral excited fragment $D^{*}$ the electron occupies a Rydberg state with quantum number $n>1$. The difference between the two FDI pathways lies in how fast the ionizing electron escapes following the turn on of the laser field [2]. In pathway A, electron 1 tunnel ionizes and escapes early on. Electron 2 gains energy from an EI-like process and tunnel ionizes. It does not have enough drift energy to escape when the laser field is turned off, and finally, it occupies a Rydberg state, $\mathrm{D}^{*}$. In pathway $\mathrm{B}$, electron 1 tunnel ionizes and quivers in the laser field, returning to the core. Electron 2 gains energy from both an EI-like process and the returning electron 1 and tunnel ionizes after a few periods of the laser field. When the laser field is turned off, electron 1 does not have enough energy to escape and remains bound in a Rydberg state. It follows that electron-electron correlation is more pronounced in pathway B [2].

To compute the FDI probability as a function of the time delay $\Delta t$ of the $\omega$ and $2 \omega$ pulses, we use

$$
P^{\mathrm{FDI}}(\Delta t)=\frac{\sum_{v, i} P_{\nu} \Gamma(\Delta t, v, i) P^{\mathrm{FDI}}(\Delta t, v, i)}{\sum_{v, i} P_{\nu} \Gamma(\Delta t, v, i)},
$$

where $i$ refers to the different orientations of the molecule with respect to the $z$ component of the laser field. We consider only two cases of planar alignment; that is, one side of the equilateral molecular triangle is either parallel or perpendicular to the $\hat{z}$ axis. $\Gamma(\Delta t, v, i)$ is given by

$$
\Gamma(\Delta t, v, i)=\int_{t_{i}}^{t_{f}} \Gamma\left(t_{0}, \Delta t, v, i\right) d t_{0},
$$

where the integration is over the duration of the OTC field. $\Gamma\left(t_{0}, \Delta t, v, i\right)$ is the ionization rate at time $t_{0}$ for a certain molecular orientation $i$, vibrational state $v$, and time delay $\Delta t . P_{v}$ is the percentage of the vibrational state $v$ in the initial state of $\mathrm{D}_{3}^{+}$[29]. $P^{\mathrm{FDI}}(\Delta t, v, i)$ is the number of FDI events out of all initiated classical trajectories for a certain molecular orientation $i$, vibrational state $v$, and time delay $\Delta t$. Due to the challenging computations involved, we approximate Eq. (2) using the $v=8$ state of $\mathrm{D}_{3}{ }^{+}$. This approximation is justified since we find that the $v=8$ state contributes the most in the sum in Eq. (2). We obtain very similar results for the $v=7,9$ states, which contribute to the sum in Eq. (2) less than the $v=8$ state but more than the other states.

\section{RESULT AND DISCUSSION}

In Fig. 1(a), for $E_{2 \omega}=0.05$ a.u., we plot the FDI probability as a function of the time delay for $\Delta t \in\left[0, T_{2 \omega}\right]$. The results are periodic with $T_{2 \omega} / 2$. We find that the FDI probability changes significantly with $\Delta t$. This change is mainly due to pathway $\mathrm{B}$ with a probability that varies from $1.2 \%$ at $\Delta t=-0.2 T_{2 \omega}$, $-0.7 T_{2 \omega}$ to $6.7 \%$ at $\Delta t=-0.4 T_{2 \omega},-0.9 T_{2 \omega}$. In contrast, the probability of pathway A changes significantly less, varying from $2.4 \%$ to $3.7 \%$. For $E_{2 \omega}<0.05$ a.u., the probability of pathway B varies less than for $E_{2 \omega}=0.05$ a.u.

Control of electron-electron correlation in double ionization in atoms has been demonstrated through the free parameters $\Delta t$ and $E_{2 \omega}$ of OTC laser fields [39-44]. The time-delay between the laser fields can significantly affect the time and the distance of the closest approach of the returning electron [11]. For FDI, this is demonstrated in Fig. 1(b). For each classical trajectory labeled as FDI, we compute the maximum of the Coulomb potential energy $1 /\left|\mathbf{r}_{1}-\mathbf{r}_{2}\right|$, $V_{12}^{\max }$. Then, we plot the distribution of $V_{12}^{\max }$ as a function of $\Delta t$. The minimum values of $V_{12}^{\max }$ correspond to electron 1 being at a maximum distance from the core, i.e., minimum electron-electron correlation. Comparing Figs. 1(a) with 1(b), we find that these minima occur at the same $\Delta t$, where the FDI probability and the probability of pathway B are minimum, i.e., at $\Delta t=-0.2 T_{2 \omega},-0.7 T_{2 \omega}$.

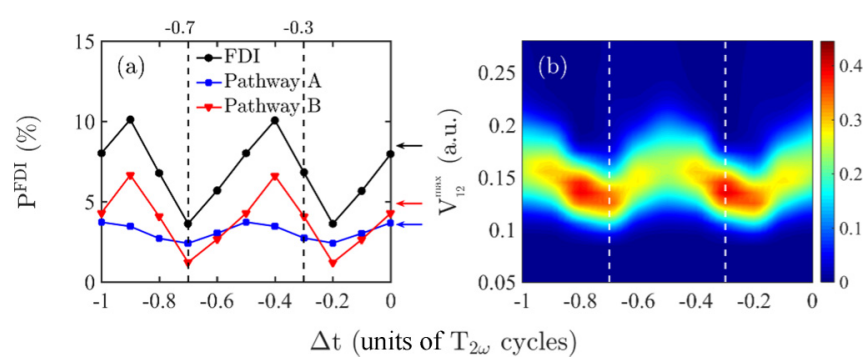

FIG. 1. (a) The FDI probability and the probabilities of pathways $\mathrm{A}$ and $\mathrm{B}$ and (b) the distribution of $V_{12}^{\max }$ are plotted as a function of $\Delta t$ for $E_{\omega}=0.08$ a.u. and $E_{2 \omega}=0.05$ a.u. In (a) the arrows on the right indicate the corresponding probabilities when $E_{2 \omega}=0$ a.u. 




FIG. 2. The distribution of $p_{z}$ for (a1) FDI and for pathways (a2) $\mathrm{A}$ and (a3) $\mathrm{B}$ is plotted as a function of $\Delta t$. For each $\Delta t$, the distribution of $p_{z}$ for FDI is normalized to 1, while for pathways A and $\mathrm{B}$ it is normalized with respect to the total FDI probability. The distribution of the time electron 1 tunnel ionizes during half cycles 1 and 2 for (b1) FDI and for pathways (b2) A and (b3) B is plotted as a function of $\Delta t$. For each $\Delta t$, the distribution of $t_{0}$ in (b1)-(b3) is normalized to $1 . t_{\max }$ is plotted with white dots (that appear as white lines) in (b2) and (b3).

The probability of each FDI pathway and $V_{12}^{\max }$ are not experimentally accessible quantities. To demonstrate the presence of electron-electron correlation in FDI, in addition to the sharp change in the FDI probability with $\Delta t$, we need one more experimentally accessible observable. This observable should bear clear signatures of the prevalence of pathway A at the $\Delta t$ where the minima of the FDI probability occur, i.e., at $\Delta t=-0.2 T_{2 \omega},-0.7 T_{2 \omega}$. We find that such an FDI observable is the change of the momentum of the escaping electron along the polarization direction of the fundamental $(\omega)$ laser field $p_{z}$ with $\Delta t$.

In Fig. 2(a1) we plot the distribution of $p_{z}$ as a function of $\Delta t$ for one period of the results, that is, in the interval $\Delta t \in$ $\left[-0.7 T_{2 \omega},-0.2 T_{2 \omega}\right]$ in steps of $\Delta t=0.1 T_{2 \omega}$. We find that the distribution of $p_{z}$ has a $\mathrm{V}$ shape. It consists of two branches that have a maximum split at $\Delta t=-0.7 T_{2 \omega}$, with peak values of $p_{z}$ around -0.85 and 0.85 a.u.. The two branches coalesce at $\Delta t=-0.3 T_{2 \omega}$, with $p_{z}$ centered around zero. Moreover, FDI events with electron 1 tunnel ionizing during half cycles with extrema at $n T_{\omega}\left(n / 2 T_{\omega}\right)$ contribute to the upper (lower) branch of the distribution of $p_{z} . n$ takes both positive and negative integer values. We find that half cycles 1 and 2 [see Figs. 3(a1) and 3(a2)], with extrema at zero and $T / 2$ of the $E_{\omega}$ laser field, respectively, contribute the most to the momentum distribution of $p_{z}$. Thus, it suffices to focus our studies on half cycles 1 and 2 .

First, we investigate the change in the distribution of the time electron 1 tunnel ionizes $t_{0}$ with $\Delta t$ [see Fig. 2(b1)]. When the second-harmonic $(2 \omega)$ field is turned off, $t_{0}$ is centered around the extrema of half cycles 1 and 2 (not shown). However, when the $2 \omega$ field is turned on, depending on $\Delta t$, electron 1 tunnel ionizes at times $t_{0}$ that are shifted to the right or to the left of the extrema of half cycles 1 and 2 [see Fig. 2(b1)]. Moreover, we find that $t_{0}$ shifts monotonically from the lowest value of the shift at $\Delta t=-0.3 T_{2 \omega}$ to its highest value at $\Delta t=-0.7 T_{2 \omega}$. We find that this change in $t_{0}$ is due to the monotonic change with $\Delta t$ of the time $t_{\max }$ when
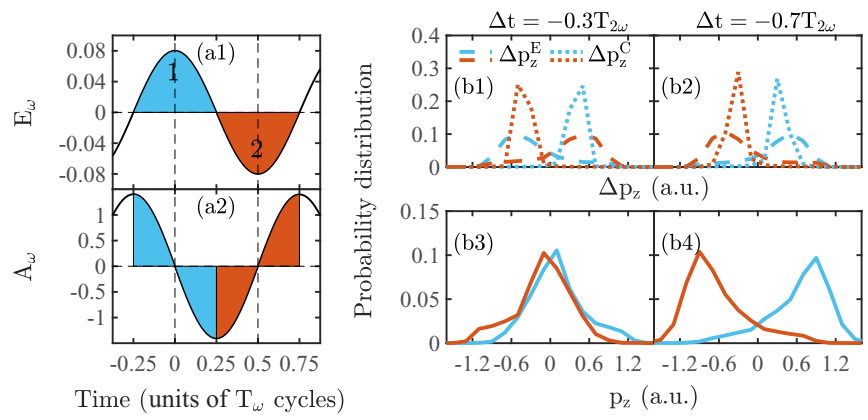

FIG. 3. Half cycles 1 and 2 for (a1) $E_{\omega}$ and (a2) its vector potential. For pathway $\mathrm{A}$, the distributions of $\Delta p_{z}^{E}$ and $\Delta p_{z}^{C}$ are plotted for half cycles 1 and 2 for (b1) $\Delta t=-0.3 T_{2 \omega}$ and (b2) $\Delta t=-0.7 T_{2 \omega}$. The distribution of $p_{z}$ is plotted for half cycles 1 and 2 for (b3) $\Delta t=-0.3 T_{2 \omega}$ and (b4) $\Delta t=-0.7 T_{2 \omega}$.

the magnitude of the OTC laser field is maximum. That is, for each $\Delta t$, we compute the time $t_{\max }$ when the laser field in Eq. (1) is maximum. $t_{\max }$ is also the time that the ionization rate is maximum. We plot $t_{\max }$ for half cycles 1 and 2 in Figs. 2(b2) and 2(b3). We compare $t_{\max }$ with the distribution of $t_{0}$ for pathways $\mathrm{A}$ and $\mathrm{B}$. We find $t_{\max }$ to be closest to the distribution of $t_{0}$ for pathway A. Indeed, only when electron 1 is the escaping electron will the time electron 1 tunnel ionizes be roughly equal to the time the ionization rate is maximum. In pathway $\mathrm{B}$ it is electron 2 that escapes. Thus, the time $t_{0}$ must be such that both the ionization rate and the electron-electron correlation efficiently combine to ionize electron 2 .

Next, for pathway A, we explain how the two brunches of the distribution of $p_{z}$ split when $t_{0}$ shifts to the right of the extrema of half cycles 1 and $2\left(\Delta t=-0.7 T_{2 \omega}\right)$ or coalesce when $t_{0}$ shifts to the left $\left(\Delta t=-0.3 T_{2 \omega}\right)$.

We compute the changes in $p_{z}$ of the escaping electron 1 due to the $\omega$ field as well as due to the interaction of electron 1 with the core. These momentum changes are given by

$$
\begin{aligned}
& \Delta p_{z}^{E}\left(\Delta t, t_{0}\right)=\int_{t_{0}}^{\infty}-E_{\omega}(t) d t \\
& \Delta p_{z}^{C}\left(\Delta t, t_{0}\right)=\int_{t_{0}}^{\infty}\left(\sum_{i=1}^{3} \frac{\mathbf{R}_{\mathbf{i}}-\mathbf{r}_{1}}{\left|\mathbf{r}_{1}-\mathbf{R}_{i}\right|^{3}}+\frac{\mathbf{r}_{1}-\mathbf{r}_{2}}{\left|\mathbf{r}_{1}-\mathbf{r}_{2}\right|^{3}}\right) \cdot \hat{z} d t
\end{aligned}
$$

with $\mathbf{R}_{i}$ being the position of the nuclei. Using the times $t_{0}$ for the events labeled as pathway $\mathrm{A}$, we plot the probability distributions of $\Delta p_{z}^{E}$ and $\Delta p_{z}^{C}$ at $\Delta t=-0.3 T_{2 \omega}$ and at $\Delta t=-0.7 T_{2 \omega}$ in Figs. 3(b1) and 3(b2), respectively. We find that, for both $\Delta t$, the distribution of $\Delta p_{z}^{C}$ peaks at positive (negative) values of $\Delta p_{z}^{C}$ when electron 1 tunnel ionizes during half cycle 1 (2). Indeed, during half cycle 1 (2), electron 1 tunnel ionizes to the left (right) of the field-lowered Coulomb potential. Then, the force from the core acts along the positive (negative) $\hat{z}$ axis, resulting in the distribution $\Delta p_{z}^{C}$ peaking around positive (negative) values for half cycle $1(2)$. We find that the contribution of the electron-electron repulsion term is small compared to the attraction from the nucleus in $\Delta p_{z}^{C}$. In contrast, the distribution of $\Delta p_{z}^{E}$ peaking at positive or negative values of $\Delta p_{z}^{E}$ depends on whether $t_{0}$ shifts to the right or to the left of the extrema of half cycles 1 and 2; 


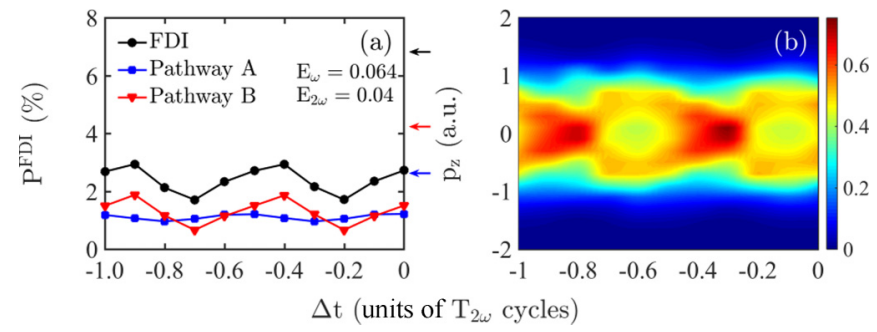

FIG. 4. (a) and (b) The same as Figs. 1(a) and 2(a1), respectively, for $\mathrm{H}_{2}$.

that is, it depends on $\Delta t$. For $\Delta t=-0.3 T_{2 \omega}$, when $t_{0}$ shifts to the left of the extrema of half cycles 1 (2), the vector potential is positive (negative), resulting in the distribution of $\Delta p_{z}^{E}$ peaking at negative (positive) values of $\Delta p_{z}^{E}$. Similarly, for $\Delta t=-0.7 T_{2 \omega}$, the distribution of $\Delta p_{z}^{E}$ peaks at positive (negative) values of $\Delta p_{z}^{E}$ for half cycle 1 (2).

In Figs. 3(b3) and 3(b4), we plot the distributions of the final momentum $p_{z}$, which is given by $\Delta p_{z}^{E}+\Delta p_{z}^{C}+p_{z, t_{0}}$. The distribution of the component of the initial momentum of electron $1 p_{z, t_{0}}$ has a small contribution to $p_{z}$ and is not shown. In Fig. 3(b3), for $\Delta t=-0.3 T_{2 \omega}$, we show that the distributions of $p_{z}$ for half cycles 1 and 2 are similar and peak at zero. They give rise to the two branches of the distribution $p_{z}$ coalescing in Figs. 2(a2) and 2(a1). In Fig. 3(b4), for $\Delta t=-0.7 T_{2 \omega}$, we find that the distributions of $p_{z}$ for half cycles 1 and 2 are quite different, with peaks at 0.85 and -0.85 a.u., respectively. They give rise to the split of the two branches of the distribution $p_{z}$ in Figs. 2(a2) and 2(a1). Unlike pathway A, for pathway B the distribution of $p_{z}$ as a function of $\Delta t$ in Fig. 2(a3) is very broad. The reason is that electron 2 has time to interact with the core since it tunnel ionizes after a few cycles of the laser field.

Finally, we show that a similar level of control of electronelectron correlation with OTC fields cannot be achieved for $\mathrm{H}_{2}$. We choose $E_{\omega}=0.064$ a.u. so that $E_{\omega}$ for $\mathrm{H}_{2}$ and $\mathrm{D}_{3}{ }^{+}$ has the same percentage difference from the field strength that corresponds to over-the-barrier ionization. We choose $E_{2 \omega}=0.04$ a.u. so that $E_{\omega} / E_{2 \omega}$ is the same for both molecules. We show in Fig. 4(a) that, for all $\Delta t$, the FDI probability significantly reduces when the $2 \omega$ field is turned on. Indeed, its maximum value is $2.7 \%$ compared to $6.8 \%$ for $E_{2 \omega}=0$ a.u. In contrast, in $\mathrm{D}_{3}{ }^{+}$the FDI probability changes from $8.5 \%$ without $2 \omega$ field to a maximum value of $10.5 \%$ for $E_{2 \omega}=0.05$ a.u. We find that the FDI probability and the probability of pathway $\mathrm{B}$ do not significantly change with $\Delta t$. In addition, the two branches of the $V$-shaped distribution $p_{z}$ of the escaping electron are not as pronounced in Fig. 4(b) as for $\mathrm{D}_{3}{ }^{+}$. The results in Fig. 4 are obtained when the internuclear axis of $\mathrm{H}_{2}$ is parallel to $E_{\omega}$. We find similar results for a perpendicular orientation; however, for $\mathrm{E}_{2 \omega}=0$ a.u., the FDI probability is almost zero. The much lower FDI probability for $\mathrm{H}_{2}$ when the $2 \omega$ laser pulse is switched on shows that the laser pulse that is perpendicularly polarized to the molecular axis drives away a large percentage of the electrons that would otherwise remain in Rydberg states if $E_{2 \omega}=0$. However, for $\mathrm{D}_{3}{ }^{+}$, this effect is counteracted by the stronger attractive force an electron experiences due to the presence of a third nucleus in a triangular configuration.

\section{CONCLUSION}

In conclusion, we have shown that control of electronelectron correlation in FDI can be achieved employing OTC fields in $\mathrm{D}_{3}{ }^{+}$. We find that the FDI probability changes sharply with the time delay between the two laser fields. Moreover, we identify a split in the distribution of the final momentum of the escaping electron that takes place at time delays where the FDI probability is minimum. We show this split to be a signature of the absence of electron-electron correlation. It then follows that electron-electron correlation is present for the time delays where the FDI probability is maximum. Future experiments can employ our scheme to demonstrate the importance of electron-electron correlation in FDI.

\section{ACKNOWLEDGMENTS}

A.E. acknowledges EPSRC Grant No. J0171831 and the use of the computational resources of Legion at UCL. M.F.K. acknowledges support from the German Research Foundation (DFG) via the Cluster of Excellence: Munich Centre for Advanced Photonics (MAP) and from the European Union (EU) via the European Research Council (ERC) grant ATTOCO.
[1] B. Manschwetus, T. Nubbemeyer, K. Gorling, G. Steinmeyer, U. Eichmann, H. Rottke, and W. Sandner, Phys. Rev. Lett. 102, 113002 (2009).

[2] A. Emmanouilidou, C. Lazarou, A. Staudte, and U. Eichmann, Phys. Rev. A 85, 011402(R) (2012).

[3] T. Nubbemeyer, K. Gorling, A. Saenz, U. Eichmann, and W. Sandner, Phys. Rev. Lett. 101, 233001 (2008).

[4] J. Yao, B. Zeng, H. Xu, G. Li, W. Chu, J. Ni, H. Zhang, S. L. Chin, Y. Cheng, and Z. Xu, Phys. Rev. A 84, 051802(R) (2011).

[5] J. McKenna, A. M. Sayler, B. Gaire, N. G. Johnson, K. D. Carnes, B. D. Esry, and I. Ben-Itzhak, Phys. Rev. Lett. 103, 103004 (2009).
[6] A. M. Sayler, J. McKenna, B. Gaire, N. G. Kling, K. D. Carnes, and I. Ben-Itzhak, Phys. Rev. A 86, 033425 (2012).

[7] J. McKenna, A. M. Sayler, B. Gaire, N. G. Kling, B. D. Esry, K. D. Carnes, and I. Ben-Itzhak, New J. Phys. 14, 103029 (2012).

[8] A. Chen, H. Price, A. Staudte, and A. Emmanouilidou, Phys. Rev. A 94, 043408 (2016).

[9] A. S. Alnaser, T. Osipov, E. P. Benis, A. Wech, B. Shan, C. L. Cocke, X. M. Tong, and C. D. Lin, Phys. Rev. Lett. 91, 163002 (2003).

[10] H. Niikura, F. Légaré, R. Hasbani, A. D. Bandrauk, M. Yu. Ivanov, D. M. Villeneuve, and P. B. Corkum, Nature (London) 417, 917 (2002).

[11] M. Kitzler and M. Lezius, Phys. Rev. Lett. 95, 253001 (2005). 
[12] M. Richter, M. Kunitski, M. Schöffler, T. Jahnke, L. Ph. H. Schmidt, and R. Dörner, Phys. Rev. A 94, 033416 (2016).

[13] S. Larimian, C. Lemell, V. Stummer, J.-W. Geng, S. Roither, D. Kartashov, L. Zhang, M.-X. Wang, Q. Gong, L.-Y. Peng, S. Yoshida, J. Burgdörfer, A. Baltuska, M. Kitzler, and X. Xie, Phys. Rev. A 96, 021403(R) (2017).

[14] D. Ray, F. He, S. De, W. Cao, H. Mashiko, P. Ranitovic, K. P. Singh, I. Znakovskaya, U. Thumm, G. G. Paulus, M. F. Kling, I. V. Litvinyuk, and C. L. Cocke, Phys. Rev. Lett. 103, 223201 (2009).

[15] H. Li, D. Ray, S. De, I. Znakovskaya, W. Cao, G. Laurent, Z. Wang, M. F. Kling, A. T. Le, and C. L. Cocke, Phys. Rev. A 84, 043429 (2011).

[16] X. Gong, P. He, Q. Song, Q. Ji, H. Pan, J. Ding, F. He, H. Zeng, and J. Wu, Phys. Rev. Lett. 113, 203001 (2014).

[17] S. De, I. Znakovskaya, D. Ray, F. Anis, N. G. Johnson, I. A. Bocharova, M. Magrakvelidze, B. D. Esry, C. L. Cocke, I. V. Litvinyuk, and M. F. Kling, Phys. Rev. Lett. 103, 153002 (2009).

[18] E. Frumker, C. T. Hebeisen, N. Kajumba, J. B. Bertrand, H. J. Wörner, M. Spanner, D. M. Villeneuve, A. Naumov, and P. B. Corkum, Phys. Rev. Lett. 109, 113901 (2012).

[19] I. Znakovskaya, M. Spanner, S. De, H. Li, D. Ray, P. Corkum, I. V. Litvinyuk, C. L. Cocke, and M. F. Kling, Phys. Rev. Lett. 112, 113005 (2014).

[20] I. J. Kim, C. M. Kim, H. T. Kim, G. H. Lee, Y. S. Lee, J. Y. Park, D. J. Cho, and C. H. Nam, Phys. Rev. Lett. 94, 243901 (2005).

[21] M. Kitzler, X. Xie, S. Roither, A. Scrinzi, and A. Baltuška, New J. Phys. 10, 025029 (2008).

[22] L. Brugnera, F. Frank, D. J. Hoffmann, R. Torres, T. Siegel, J. G. Underwood, E. Springate, C. Froud, E. I. C. Turcu, J. W. G. Tisch, and J. P. Marangos, Opt. Lett. 35, 3994 (2010).

[23] L. Brugnera, D. J. Hoffmann, T. Siegel, F. Frank, A. Zaïr, J. W. G. Tisch, and J. P. Marangos, Phys. Rev. Lett. 107, 153902 (2011).

[24] N. Dudovich, O. Smirnova, J. Levesque, Y. Mairesse, M. Yu. Ivanov, D. M. Villeneuve, and P. B. Corkum, Nat. Phys. 2, 781 (2006).

[25] H. Niikura, N. Dudovich, D. M. Villeneuve, and P. B. Corkum, Phys. Rev. Lett. 105, 053003 (2010).

[26] D. Shafir, Y. Mairesse, D. M. Villeneuve, P. B. Corkum, and N. Dudovich, Nat. Phys. 5, 412 (2009).

[27] C. Lefebvre, H. Z. Lu, S. Chelkowski, and A. D. Bandrauk, Phys. Rev. A 89, 023403 (2014).
[28] E. Lötstedt, T. Kato, and K. Yamanouchi, Phys. Rev. Lett. 106, 203001 (2011).

[29] V. G. Anicich and J. H. Futrell, Int. J. Mass Spectrom. Ion Proces. 55, 189 (1984).

[30] T. Ergler, B. Feuerstein, A. Rudenko, K. Zrost, C. D. Schröter, R. Moshammer, and J. Ullrich, Phys. Rev. Lett. 97, 103004 (2006).

[31] E. Goll, G. Wunner, and A. Saenz, Phys. Rev. Lett. 97, 103003 (2006).

[32] D. Talbi and R. P. Saxon, J. Chem. Phys. 89, 2235 (1988).

[33] H. Price, C. Lazarou, and A. Emmanouilidou, Phys. Rev. A 90, 053419 (2014).

[34] R. Murray, M. Spanner, S. Patchkovskii, and M. Yu. Ivanov, Phys. Rev. Lett. 106, 173001 (2011).

[35] R. Y. Rubinstein and D. P. Froese, Simulation and the Monte Carlo Method, 2nd ed. (Wiley, Hoboken, NJ, 2007).

[36] M. V. Ammosov, N. B. Delete, and V. P. Krainov, Sov. Phys. JETP 64, 1191 (1986).

[37] A. Chen, C. Lazaro, H. Price, and A. Emmanouilidou, J. Phys. B 49, 235001 (2016).

[38] T. Zuo and A. D. Bandrauk, Phys. Rev. A 52, R2511(R) (1995); T. Seideman, M. Yu. Ivanov, and P. B. Corkum, Phys. Rev. Lett. 75, 2819 (1995); D. M. Villeneuve, M. Yu. Ivanov, and P. B. Corkum, Phys. Rev. A 54, 736 (1996); E. Dehghanian, A. D. Bandrauk, and G. L. Kamta, ibid. 81, 061403(R) (2010).

[39] Y. Zhou, Q. Liao, Q. Zhang, W. Hong, and P. Lu, Opt. Express 18, 632 (2010).

[40] Y. Zhou, C. Huang, A. Tong, Q. Liao, and P. Lu, Opt. Express 19, 2301 (2011).

[41] L. Chen, Y. Zhou, C. Huang, Q. Zhang, and P. Lu, Phys. Rev. A 88, 043425 (2013).

[42] L. Zhang, X. Xie, S. Roither, Y. Zhou, P. Lu, D. Kartashov, M. Schöffler, D. Shafir, P. B. Corkum, A. Baltuška, A. Staudte, and M. Kitzler, Phys. Rev. Lett. 112, 193002 (2014).

[43] C. A. Mancuso, K. M. Dorney, D. D. Hickstein, J. L. Chaloupka, J. L. Ellis, F. J. Dollar, R. Knut, P. Grychtol, D. Zusin, C. Gentry, M. Gopalakrishnan, H. C. Kapteyn, and M. M. Murnane, Phys. Rev. Lett. 117, 133201 (2016).

[44] S. Eckart, M. Richter, M. Kunitski, A. Hartung, J. Rist, K. Henrichs, N. Schlott, H. Kang, T. Bauer, H. Sann, L. Ph. H. Schmidt, M. Schöffler, T. Jahnke, and R. Dörner, Phys. Rev. Lett. 117, 133202 (2016). 